\title{
Endomeriosis of Cesarean Section Why on Leet side? \\ ${ }^{1}$ Ali Hl Abbud, ${ }^{2}$ Ridha J Al-Basri ${ }^{3}$ Abeer H Dekan \\ ${ }^{4}$, Sarmad J Shehatha
}

ABSTRACT

\begin{abstract}
Background: Endometriosis is defined as the presence or growth of ectopic endometrial tissue outside their usual site ( the uterus). It is a common condition in women. It may occur in the ovaries, fallopian tubes, vagina and rarely, endometriosis may occur in the abdomen and lungs. Endometriosis is common among women of reproductive age. It is either primary or secondary. The triad of diagnosis is a pain with menstruation, cesarean scar and a mass in the scar. Methods: A report a series of 65 patients in the period from $1^{\text {st }}$ Jan 2013 to $31^{\text {st }}$ Dec 2017. The study was performed in Al-Kindi Teaching Hospital and a private hospital. The mean age was 35 years (range 20-45).All patients were treated surgically i.e. excision of the tumor and old surgical scar in the lower abdomen.

Result: mean age was 35 years (range 20-45). Thirty patients $(46.15 \%)$ were housewives while $35(53.84 \%)$ were officers. The interesting finding is that 52 patients had the mass in the left side, 10 in the middle and only 3 in the right side of the scar respectively.
\end{abstract}

Conclusion: Our study demonstrated a lateral asymmetry in the location of scar endometriosis i.e. mostly on the left side. The prevalence on left side of scar can be added to the triad of diagnosis mentioned above I.e. cyclical pain, cesarean scar and mass mostly in left side of the scar.

Key words: endometriosis, Cyclical pain, cesarean scar, mass in cesarean scar

${ }^{1}$ Corresponding author :Specialist surgeon, Department of surgery, Al-Kindi teaching hospital, Baghdad, Iraq Email : aliAlsaraj63@yahoo.com

${ }^{2}$ Consultant surgeon, Department of surgery, Al-Kindi teaching hospital, Baghdad, Iraq

Email : redagawad@gmail .com

${ }^{3}$ Obstetrician \& gynecologest Arbic Board, Baghdad teaching hospital, Baghdad, Iraq

Email :abeer.Gh.d@gmail.com

${ }^{4}$ Specialist surgeon, Department of surgery, Al-Kindi teaching hospital, Baghdad,

Received at128/4/2019

Accepted at 26/6/2019

\section{INTRODUCTION}

Endometriosis is defined as the presence or growth of ectopic endometrial tissue outside their usual site ( the uterus) ${ }^{(1)}$. It is a common condition in women. It may occur in the ovaries, fallopian tubes, vagina and rarely, endometriosis may occur in the abdomen and lungs. Endometriosis is common among women of reproductive age and an estimated $11 \%$ of women in the United States may have endometriosis. Women without prior pregnancies, with a family history of endometriosis, and with abnormal menstrual periods are more likely to have endometriosis $^{(2)}$. It can have a significant impact on quality of life and on the lives of partners. Symptoms include painful periods, deep pain during sex, chronic pelvic pain, painful bowel movements and urination, and premenstrual symptoms with or without abnormal bleeding and pain. Chronic fatigue, depression, infertility, cyclical lump, and back, leg and chest pain are also common symptoms ${ }^{(3)}$. The majority of the scar endometriosis have been reported after obstetrical, or gynecological procedures such as cesarean delivery, hysterectomy, episiotomy, and tubal ligations but few case reports are following appendicectomy, in the laparoscopic trocar tract, amniocentesis needle tract $^{(4-6)}$.

It is classified in 2 types, primary and secondary. The primary form of rectus abdominus is not due to previous surgical procedure and it is very rare where only 18 cases were recorded from 1984 to $2004^{(7-}$ ${ }^{8)}$. The secondary form is much more common than primary and it is usually due to obstetrical or gynecological procedures such as cesarean delivery, few due to other surgical procedures ${ }^{(9)}$.

The pathogenesis of endometriosis still remains controversial: immune, hormonal, genetic, and environmental factors may be all involved, and several theories have been proposed to explain it. Accumulating evidence suggests that during postpubertal age, under the influence of different stimuli, these misplaced and quiescent ectopic endometrial cells could acquire new phenotype, biological functions, and 
immunogenicity. So, these kinds of cells may differentiate, specializing in epithelium, glands, and stroma to form a functional ectopic endometrial tissue ${ }^{(10)}$. Endometriosis most often affects the ovaries (up to $88 \%$ of all cases), peritoneal surface, uterine ligaments, fallopian tubes, rectum, and the cervical-vaginal region ${ }^{(11)}$. Risk factors for endometriosis include early menarche, short menstrual cycle, prolonged/heavy menstrual flow, delay of first birth, reduced number of pregnancies, positive family history, obstruction of menstrual outflow ${ }^{(12)}$, exposure to diethylstilbestrol in utero ${ }^{(13)}$, prolonged exposure to endogenous estrogen, short menstrual cycles, low birth weight ${ }^{(14)}$, and exposure to endocrine-disrupting chemicals ${ }^{! 15)}$. Twin and family studies suggest a genetic component ${ }^{(15)}$. Consumption of red meat and fats is associated with an increased risk of laparoscopically confirmed endometriosis, and eating fruits, green vegetables, and $\mathrm{n}-3$ long-chain fatty acids is associated with a decreased risk (16). Prolonged lactation and multiple pregnancies are protective. Endometriosis is associated with increased risks of autoimmune diseases and ovarian endometrioid and clear-cell cancers, as well as other cancers, including non-Hodgkin's lymphoma and melanoma ${ }^{(17)}$. Signs \& symptoms include pelvic pain due to endometriosis is usually chronic (lasting $\geq 6$ months) and is associated with dysmenorrhea (in 50 to $90 \%$ of cases), dyspareunia, deep pelvic pain, and lower abdominal pain with or without back and loin pain. The pain can occur unpredictably and intermittently throughout the menstrual cycle or it can be continuous, and it can be dull, throbbing, or sharp, and exacerbated by physical acitivity. Bladder- and bowel-associated symptoms (nausea, distention, and early satiety) are typically cyclic. Pain often worsens over time and may change in character; infrequently, women report burning or hypersensitivity, symptoms that are suggestive of a neuropathic component. Symptoms overlap with those of several other gynecologic conditions (e.g., pelvic inflammatory disease, pelvic adhesions, ovarian cysts or masses, leiomyomata, and adenomyosis) and nongynecologic conditions and factors (e.g., irritable bowel syndrome, inflammatory bowel disease, interstitial cystitis, myofascial pain, depression, and a history of sexual abuse), making diagnosis challenging ${ }^{(18)}$.

There are three treatment options available: pain management, hormone treatment and surgery. The recommended first-line option is a three-month trial of paracetamol or an NSAID, either alone or in combination. If this is not effective, the next option is other (unspecified) forms of pain management and referral for assessment ${ }^{(19)}$. NICE refers to its guideline on drug treatment of neuropathic pain in non-specialist settings which recommends a choice of amitriptyline, duloxetine, gabapentin or pregabalin as initial treatment.Hormonal treatment such as the combined oral contraceptive pill or a progestogen should be offered to women with suspected, confirmed or recurrent endometriosis. Surgery should preferably be per-formed laparoscopically. Excision should be considered for endometriomas. Hormonal treatment should be considered after laparoscopic excision or ablation of endometriosis to prolong the benefits of surgery and manage symp-toms. When hysterectomy is an option it should, in the absence of contraindica-tions, be carried out laparoscopically when combined with surgical treatment of endometriosis ${ }^{(20)}$.

\section{METHODS}

We report a series of sixty five (65) patients in the period from $1^{\text {st }}$ Jan 2013 to $31^{\text {st }}$ Dec 2017. The study was performed in Al-Kindi Teaching Hospital and a private hospital. The mean age was 35 years (range 20-45).All patients were treated surgically i.e. excision of the tumor and old surgical scar in the lower abdomen.

Inclusion criteria are :

1. Females of child bearing age

2. Patients with a triad of periodic pain associated with menses, history of cesarean section and a mass in the scar.

3. Patients were treated surgically.

4. Mass proved by histopathology to be endometriosis.

Exclusion criteria :

1. Patients were at menopause age. 
2. Patients took medical therapy.

3. Endometriosis in sites other than cesarean section.

All specimen were send for histopathological examination to prove the diagnosis of endometriosis. Patients were kept in hospital for 2 days postoperatively and discharged on the $3^{\text {rd }}$ day on intramuscular antibiotics (Ceftriaxon 1gm) for 2 days. Oral analgesia was given to be taken on need ( Iboprofen $200 \mathrm{mg}$ ). They were instructed to come back 5 days from discharge for suture removal. All patients were followed for 6 months after surgery. The aim of the study is to report our finding that most of the masses were in the left side of the cesarean scar.

\section{RESULTS}

The study includes 65 patients. Mean age was 35 years (range 20-45). Thirty patients $(46.15 \%)$ were housewives while $35(53.84 \%)$ were officers. Table 1 shows the demography of the patients.
All patients were presenting with triad of periodic pain associated with menstrual cycle and scar of a previous cesarean section. The pain was moderate to severe needing analgesia to relief the pain in most patients. Ten patients were not aware to the presence of a mass only bothered by the cyclic pain. The interesting finding is that 52 patients had the mass in the left side, 10 in the middle and only 3 in the right side of the scar respectively. See table-2.

When patients were attended our outpatient clinic no treatment was prescribed, because most patients tried treatment with no benefit, and they were seeking surgery to terminate their compliant. The surgery was to excise the scar and complete excision of the mass.

All the specimen were send for histopathological examination. All were proved to be endometriosis From table-3 we see that all patients received analgesia, 60 patients received hormonal therapy. Fifty of them received oral contraceptives and 10 Aromatase inhibitors.

Table-1 Demography of patients

\begin{tabular}{|l|l|l|l|}
\hline Age (years) & No. of patients $(\%)$ & No. of housewife $(\%)$ & No. of officers (\%) \\
\hline $20-29$ & $20(30.76)$ & $10(15.38)$ & $10(15.38)$ \\
\hline $30-39$ & $25(38.46)$ & $5(07.69)$ & $20(30.76)$ \\
\hline $40-45$ & $20(30.76)$ & $15(23.07)$ & $5(07.69)$ \\
\hline Total & $65(99.98)$ & $30(46.15)$ & $35(53.84)$ \\
\hline
\end{tabular}

Table -2 Details of clinical findings.

\begin{tabular}{|c|c|l|}
\hline Parameter & No. of patients (\%) & Notes \\
\hline $\begin{array}{c}\text { Pain mild } \\
\text { moderate } \\
\text { severe }\end{array}$ & $0(0)$ & $\begin{array}{l}\text { The pain was moderate to severe } \\
\text { (most common) }\end{array}$ \\
\hline Scar & $40(38.46)$ & \\
\hline Mass aware & $65(100)$ & \\
Not aware & $55(84.61)$ & Some patients were not aware to \\
the mass (only pain)
\end{tabular}


Table -3. Medical therapy before attending our clinic

\begin{tabular}{|l|l|l|}
\hline Drugs & No. of patients (\%) & \multicolumn{1}{|c|}{ Notes } \\
\hline Analgesia & $65(100)$ & Oral ibuprofen/Diclofenac inj. \\
\hline oral contraceptive pill & $50(76.92)$ & Some discontinued treatment \\
\hline Aromatase inhibitors & $10(15.38)$ & No details available \\
\hline
\end{tabular}

\section{DISCUSSION}

Scar endometriosis patients are often referred to the general surgeons because the clinical presentation suggests a surgical cause. In a study by Blanco et al ${ }^{(9)}$ the diagnosis was initially confused with an abscess, hematoma, suture granuloma, inguinal hernia, incisional hernia, and abdominal wall tumor.This entity can result in unnecessary procedures, delayed or misdiagnosis, and can cause emotional and physical distress to the patient. So it is important for the attended doctor to take good detailed clinical, surgical, menstrual and drug history. The patients should be fully examined. Great attention should be paid to the triad of cyclic pain during menses, scar of cesarean section and lump in the scar. Non of the authors in literature mentioned the site of the tumor in the cesarean scar. We claim that this is the first time to attract attention that left side of the scar is the commonest site for endometriosis mass. But Paolo $\mathrm{V}$ et al found in their large surgical series that similar to ovarian endometriosis, ureteral disease is observed more frequently on the left than the right side. Interestingly, the proportion of left-sided gonadal and ureteral lesions is remarkably similar $(63 \%$ and $64 \%)^{(21)}$. Almost two-thirds of the patients had ureteral lesions on the lefthand side. This is compatible with the menstrual reflux theory and with the anatomical differences of the left and right hemipelvis This predilection of left sided gonadal endometriosis may partly explain our finding. Rapture and implantation of endometrial cells in left side will be the result. The asymmetry of both ovarian and ureteral forms may be the expression of a common underlying anatomical condition that facilitates adhesion and growth of endometrial cells on the left pelvic side wall $^{(22-23)}$.

We can add to the previous causes that nurses may contribute since they stand on left side. Contamination of their gloves with endometrial cells may cause implantation of them in the wound, hence prevalence on left side.

\section{CONCLUSION}

Our study demonstrated a lateral asymmetry in the location of scar endometriosis. This is compatible with the anatomical differences of the left and right hemipelvis, is in line with epidemiological data suggesting that the major pathogenetic mechanism of peritoneal and ovarian endometriosis is not substantially different, and supports the menstrual reflux theory. We can add to these the role of nursing staff for implantation of endometrial cells. The prevalence on left side of scar can be added to the triad of diagnosis mentioned above I.e. cyclical pain, cesarean scar and mass mostly in left side of the scar.

\section{REFRENCES}

1. Gupta P, Gupta S, Acta Med Iran. 2015;53(12):793-795

2. Alan N. Peiris, Ellen Chaljub, Dillon Medlock, JAMA. 2018;320(24):2608.

3. Dean, Erin ,Primary Health Care.2017; 27(9): 15-16.

4. Padmanabhan LD, Mhaskar R, Mhaskar A. Scar endometriosis. J Obstet Gynaecol India 2013;53(1):59-61.

5. Bhowmick RN, Paul P, Dutta $S$, et al. Endometriosis of laparotomy scar. J Obstet Gynaecol India 2016;36:130-2.

6. Chatterjee SK. Scar endometriosis: A Clinicopathological study of 17 cases. Obstet Gynecol 2011;56(1):81-4.

7. Coley BD, Casola G. Incisional Endomerioma of Rectu Abdominis and subcutaneous tissue: CT appearance. Am J Roentgel. 2012; 160:549-50

8. Feeney $\mathrm{J}$ etal, Endomeriona of the rectus sheath after Caesarean Section. Can Assoc Radiol J. 2008; 59:210-12. 
9. Blanco RG, Parithivel VS, Shah AK, et al. Abdominal wall endometriomas. Am J Surg 2013;185(6):596-8.

10. A.S. Laganà, E. Sturlese, G. Retto, V. Sofo, O. Triolo Interplay between misplaced Müllerian-derived stem cells and peritoneal immune dysregulation in the pathogenesis of endometriosis Obstet Gynecol Int, 2013 (2013), p. 527041.

11. S. Butticè, A.S. Laganà, V. Barresi, et al. Lumbar ureteral stenosis due to endometriosis: our experience and review of the literature Case Rep Urol, 2013; vol13: p. 812-15.

12. Sanfilippo JS, Wakim NG, Schikler KN, Yussman MA. Endometriosis in association with uterine anomaly. Am J Obstet Gynecol 2016;154:39-43.

13. Missmer SA, Hankinson SE, Spiegelman D, Barbieri RL, Michels KB, Hunter DJ. In utero exposures and the incidence of endometriosis. Fertil Steril 2014;82:15011508 .

14. Missmer SA, Hankinson SE, Spiegelman D, Barbieri RL, Marshall LM, Hunter DJ. Incidence of laparoscopically confirmed endometriosis by demographic, anthropometric, and lifestyle factors. Am J Epidemiol 2014;160:784-796.

15. Diamanti-Kandarakis E, Bourguignon JP, Giudice LC, et al. Endocrine-disrupting chemicals: an Endocrine Society scientific statement. Endocr Rev 2009;30:293-342.
16. Montgomery GW, Nyholt DR, Zhao ZZ, et al. The search for genes contributing to endometriosis risk. Hum Reprod Update 2008; 14:447-457.

17. Goldstein DP, deCholnoky C, Emans SJ, Leventhal JM. Laparoscopy in the diagnosis and management of pelvic pain in adolescents. J Reprod Med 2015;24:251-256.

18. SusannahHoggSanjayVyas. Endometriosis. Obstetrics, Gynaecology \& Reproductive Medicine 2015; 25( 5: 133-141.

19. Chaplin S. Diagnosis and management of endometriosis. Prescriber 2018; 29 (3):29-30.

20. National Institute for Health and Care Excellence. Fertility problems: assessment and treatment. CG156. February 2013 (Updated September 2017). Available from: https://www.nice.org.uk/guidance/cg156.

21. Paolo V, Anna P, Antonella P, Sarah V, Giovanna S, Pier G C. Is ureteral endometriosis an asymmetric disease?. Int $\mathrm{J}$ Gynaec \& Obset 2000; 107(4): 559-61.

22. Vercellini, P, Aimi, G, Giorgi, O, Maddalena, S, Carinelli, S, Crosig-nani, PG. Is cystic ovarian endometriosis an asymmetric disease Br J Obstet Gynaecol 1998; 105: 1018- 1021.

23. Vercellini, P, Pisacreta, A, Vicentini, S, Stellato, G, Pesole, A, Crosignani, PG. Lateral distribution of nonendometriotic ovarian cysts. Br J Obstet Gynaecol 2000; 107: 556- 558. 